\title{
Análisis de indicadores bioquímicos en un ataifor almohade
}

\author{
María Luisa Cañabate Guerrero* \\ Alberto Sánchez Vizcaíno**
}

\section{INTRODUCCIÓN}

El estudio de los indicadores bioquímicos de contenidos en recipientes cerámicos engloba el análisis de una serie de compuestos orgánicos, cuya identificación puede ayudar en la determinación del posible uso/s de un recipiente. A través de su estudio, y en conjunción con el resto de los datos arqueológicos disponibles, se podría llegar a precisar y/o establecer por una parte, el tipo de contenido y como consecuencia una aproximación a la dieta (CONDAMIN et al., 1976; NEEDHAM AND EVANS, 1987; MICHEL et al., 1993; EVERSHED, 1993; CHARTERS et al., 1995); y por otra, en un plano más general, una definición y delimitación más completa de las áreas de actividad (RÍ́sQUEZ et al., 1995; SÁNCHEZ et al., 1995; CAÑABATE Y SÁNCHEZ, en prensa).

Entre los indicadores que en la actualidad están siendo objeto de estudio deben destacarse principalmente los lípidos, cuya presencia habitual en diversos alimentos y sus características químicas (EVERSHED, 1993), los hacen idóneos para la identificación de contenidos. Dentro del grupo de los lípidos se engloban una serie de compuestos con diferente estructura química, que tienen en común el ser insolubles en agua y solubles en disolventes orgánicos. El conocimiento de sus características y propiedades químicas, así como de los procesos degradativos que les pueden afectar (EVERSHED et al., 1992), constituye un paso previo a cualquier investigación que tenga a los lípidos como protagonistas.

Los compuestos que dentro del grupo de los lípidos tienen interés arqueológico son: ácidos grasos, acilgliceroles, esteroles, ésteres de ceras, alcanos y alcoholes de cadena larga. Cada uno de estos grupos de compuestos, en función de la intensidad con que se detecten, pueden estar indicando un tipo determinado de contenido. Así mismo, la detección de compuestos que en la naturaleza son excluyentes puede llamar la atención sobre la presencia de una mezcla de diferentes sustancias (CHARTERS et al., 1995), o bien, una reutilización del recipiente.

En este trabajo presentamos los resultados del análisis realizado sobre un ataifor almohade procedente del Castillo de Sabiote (Jaén) con una cronología de finales del siglo Xll-principios del siglo XIII (Foto I). Los análisis químicos han sido llevados a cabo en el Departamento de Geoquímica Orgánica de la Universidad de Bristol (Reino Unido).

\section{MATERIALES Y MÉTODOS}

Preparación de la muestra, extracción y análisis.

El procedimiento de análisis empleado sigue el protocolo desarrollado por el Dr. Richard P. Evershed en la Universidad de Bristol (EVERSHED, 1990). Tras limpiar el fragmento de la tierra adherida a él, se recuperan mediante raspado dos gramos de muestra de su interior. Normalmente el proceso consiste en tomar un trozo del fragmento, limpiarlo y machacarlo pero, en este caso, al tratarse de un recipiente completo y en un excelente estado de conservación, se ha procedido a tomar la muestra raspando con cuidado en el interior. La muestra es entonces almacenada a $-40^{\circ} \mathrm{C}$, hasta el momento de su análisis.

* Dpto. de Territorio y Patrimonio histórico. Áreas de Prehistoria y Arqueología. Universidad de Jaén. 
El paso siguiente a la toma de muestra consiste en la extracción. Para ello primero se añade a la muestra ya pulverizada $20 \mu \mathrm{l}$ de standard interno (n-tetratriacontano, en un

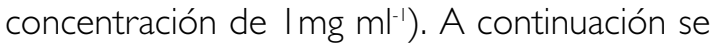
añade la mezcla extractante (cloroformo:metanol; 2: I, I0 ml) y se realizan dos extracciones de 15 min. en un baño de ultrasonidos. La mezcla extraída se centrifuga (3500 r.p.m. durante aproximadamente 15 min.) y el líquido sobrenadante se transfiere a un pequeño vial y se evapora el disolvente en corriente de nitrógeno. El extracto lipídico total se disuelve de nuevo en $500 \mu \mathrm{l}$ de la disolución de cloroformo:metanol y se toma una alícuota de 100 $\mu$ l para proceder al análisis.

\section{Derivatización.}

El extracto lipídico se derivatiza por tratamiento de la alícuota $\left(20 \mu \mathrm{l} ;\right.$ a $60^{\circ} \mathrm{C}$; durante $20 \mathrm{~min}$ ) con N,O-bis (trimetilsilil) trifluoroacetamida (BSTFA) que contiene $1 \% \mathrm{v} / \mathrm{v}$ de trimetilclorosilano. La muestra ya derivatizada se diluye con ciclohexano y se analiza por Cromatografía de Gases y Cromatografía de Gases-Espectrometría de Masas.

\section{Cromatografía de Gases, Cromatografía de Gases-Espectrometría de Masas.}

Para la Cromatografía de Gases se emplea un cromatógrafo Hewlett-Packard 5890A, empleando un inyector on-column. La columna empleada es de alta temperatura BP-I de $15 \mathrm{~m} \times 0.2 \mathrm{~mm}$. El programa de temperatura empleado comienza a $50^{\circ} \mathrm{C}$ durante 2 min y alcanza los $350^{\circ} \mathrm{C}$ a razón de $10^{\circ} \mathrm{C} / \mathrm{min}$, manteniéndose al final el horno a $350^{\circ} \mathrm{C}$ durante 10 min. Para la Cromatografía de GasesEspectrometría de Masas, se emplea un equipo Pye Unicam 204 GC equipado con un inyector on-column. El cromatógrafo se une a un espectrómetro de masas VG 7070H.

\section{RESULTADOS}

El análisis realizado por Cromatografía de Gases del ataifor presenta el cromatograma que se muestra en la Figura I. En una primera aproximación, lo que primero se desprende del estudio de este cromatograma es la baja proporción en la que se encuentran los componentes detectados. Este hecho ya se ha observado en los análisis realizados sobre otras muestras cerámicas (aunque no de la misma época) del sur de la península ibérica (CAÑABATE Y SÁNCHEZ, en prensa). En los análisis realizados a muestras procedentes de zonas con climas húmedos, la proporción en la que se extraen los distintos componentes lipídicos es superior. No obstante, este hecho ha de ser estudiado y analizado para intentar descubrir si condiciones medioambientales tan diferentes pueden afectar de esa manera a la conservación de estas sustancias orgánicas. Otra posible causa que puede afectar a la baja recuperación de los componentes lipídicos podría ser la forma en la que se ha realizado la toma de la muestra, ya que sólo se ha podido extraer la parte superficial del recipiente.

De todos modos se puede considerar que los compuestos extraídos ofrecen una buena información acerca del posible contenido de este recipiente. Un recipiente al que por su tipología se le supone un uso como contenedor de algún tipo de líquido o sustancia generalmente no sólida.

La identificación de los picos señalados en el cromatograma, realizada a través de la Cromatografía de Gases-Espectrometría de Masas, ha diferenciado varios grupos de compuestos: ésteres de ceras, alcanos, alcoholes de cadena larga, esteroles y, en baja proporción, ácidos grasos de 16 y 18 átomos de carbono y colesterol. La presencia de todos ellos nos lleva a plantear varias cuestiones:

a.- La presencia de los ésteres de ceras (en este caso, ésteres de ceras del ácido palmítico), constituyentes importantes en la composición de la cera de abeja (CHARTERS et al., 1995), indicaría que el recipiente ha sido, bien impermeabilizado para hacerlo apto como contenedor de algún liquido, bien empleado como contenedor de la cera propiamende dicha. Así mismo, la presencia de alcanos, compuestos típicos, aunque no exclusivos de la cera de abeja, reforzaría la existencia de ésta. Finalmente, la aparición de alcoholes de cade- 
na larga sería el resultado de los procesos degradativos que sufren los ésteres de ceras, dando lugar al ácido palmítico y al alcohol de cadena larga correspondiente.

b.- Es importante comentar un tipo de indicadores de gran importancia en el estudio de contenidos, los esteroles. Los esteroles son alcoholes de alto peso molecular presentes minoritariamente en la fracción insaponificable de las grasas. El colesterol es el esterol más abundante en los animales, está presente en las células y tiene muchas e importantes funciones biológicas. Por el contrario, el campesterol y el sitosterol, son los esteroles más abundantes en las plantas. En el cromatograma de la muestra en estudio, han sido detectados tanto colesterol como sitosterol. En ambos casos resulta extraño el que no se hayan encontrado en gran concentración ácidos grasos de entre 14 y 18 átomos de carbono. Por otra parte, los pequeños picos que aparecen después de los correspondientes a los ésteres de ceras, podrían tratarse de triacilgliceroles, pero sus identidades no han podido ser determinadas debido a que sus espectros de masas no han podido ser registrados.

En conclusión, la presencia de los citados compuestos, a saber, ésteres de ceras, colesterol y sitosterol, en principio excluyentes entre sí y por tanto indicadores de contenidos diferentes, llevaría a plantear que el recipiente que se ha analizado presenta una función doméstica con múltiples usos.

\section{AGRADECIMIENTOS}

Queremos mostrar nuestro agradecimiento al Dr. R.P. Evershed de la Universidad de Bristol, así como a Ms. Stephany Dudd y Ms. Stephany Charters por su inestimable ayuda.

\section{RESUMEN}

El análisis de contenidos en recipientes supone una importante información adicional que completa el estudio de un recipiente arqueológico. En este trabajo se muestra el estudio realizado sobre un ataifor almohade procedente del Castillo de Sabiote (Jaén).

PALABRAS CLAVE: Indicadores Bioquímicos, Contenidos, Cerámica Almohade, Cromatografía de Gases, Espectrometría de Masas

\section{BIBLIOGRAFÍA}

CAÑABATE, M.L. y SÁNCHEZ, A. (1995): "Aspectos metodológicos en el análisis de indicadores bioquímicos y su aplicación al campo arqueológico". Cuadernos de Prehistoria de la Universidad de Granada (En prensa).

CHARTERS, S., EVERSHED, R.P., BLINKHORN, P.W. and DENHAM V. (1995): "Evidence for the mixing of fats and waxes in archaeological ceramics". Archaeometry 37, (I), pp. I I3-127.

CONDAMIN, J.,FORMENTI, F., METAIS, M.O., MICHEL, M. and BLOND, P. (1976): "The application of gas chromatography to the tracing of oil in ancient amphorae". Archaeometry 18, pp. 195-201.

EVERSHED, R.P. (1993): "Biomolecular archaeology and lipids". World Archaeology 25, (I), pp. 74-93.

EVERSHED, R.P., HERON, C. and GOAD, J. (1990): "Analysis of organic residues of archaeological origin by high-temperature gas chromatography and gas chromatography-mass spectrometry". Analyst I 15, (10), pp. 1339-1342.

EVERSHED, R.P.; HERON, G.; CHARTERS, S. and GOAD, L.J. (1992): "The survival of food residues: new methods of analysis, interpretation and application". Proceedings of the British Academy 77, pp. 187-208.

MICHEL, R.H., MCGOVERN, P.E., AND BADLER V.R (1993): "The first wine and beer. Chemical detection of ancient fermented beverages". Analytical Chemistry 65, (8), pp. 408-4I3.

NEEDHAM, S. and EVANS, J. (1987): "Honey and dripping; Neolithic food residues from Runnymede Bridge". Oxford Journal of Archaeology 6, (1), pp. 21-28.

RISQUEZ, C., SÁNCHEZ, A. y CAÑABATE, M.L. (1995): "Contexto, procedencia y contenido a partir de nuevas técnicas de análisis: un conjunto de ánforas de Puente Tablas (Jaén)". I Congreso de Arqueología Peninsular. Actas VII. Trabalhos de Antropologia e Etnologia 35, (3), pp. 353-376.

SÁNCHEZ, A., CAÑABATE, M.L. Y LIZCANO, R. (1996): "Phosphorus analysis at archaeological sites: an optimization of the method and interpretation of the results". Archaeometry 38, (I).

\section{ABSTRACT}

The analysis of contents in vessels contributes with additional information to complete the study on pottery. A study about an ataifor (Almohade period) from the Castle of Sabiote (Jaén, Spain) is shown in this paper.

KEY WORDS: Biochemical Markers, Contents, Almohade Ceramics, Gas Chromatography, Mass Spectrometry 


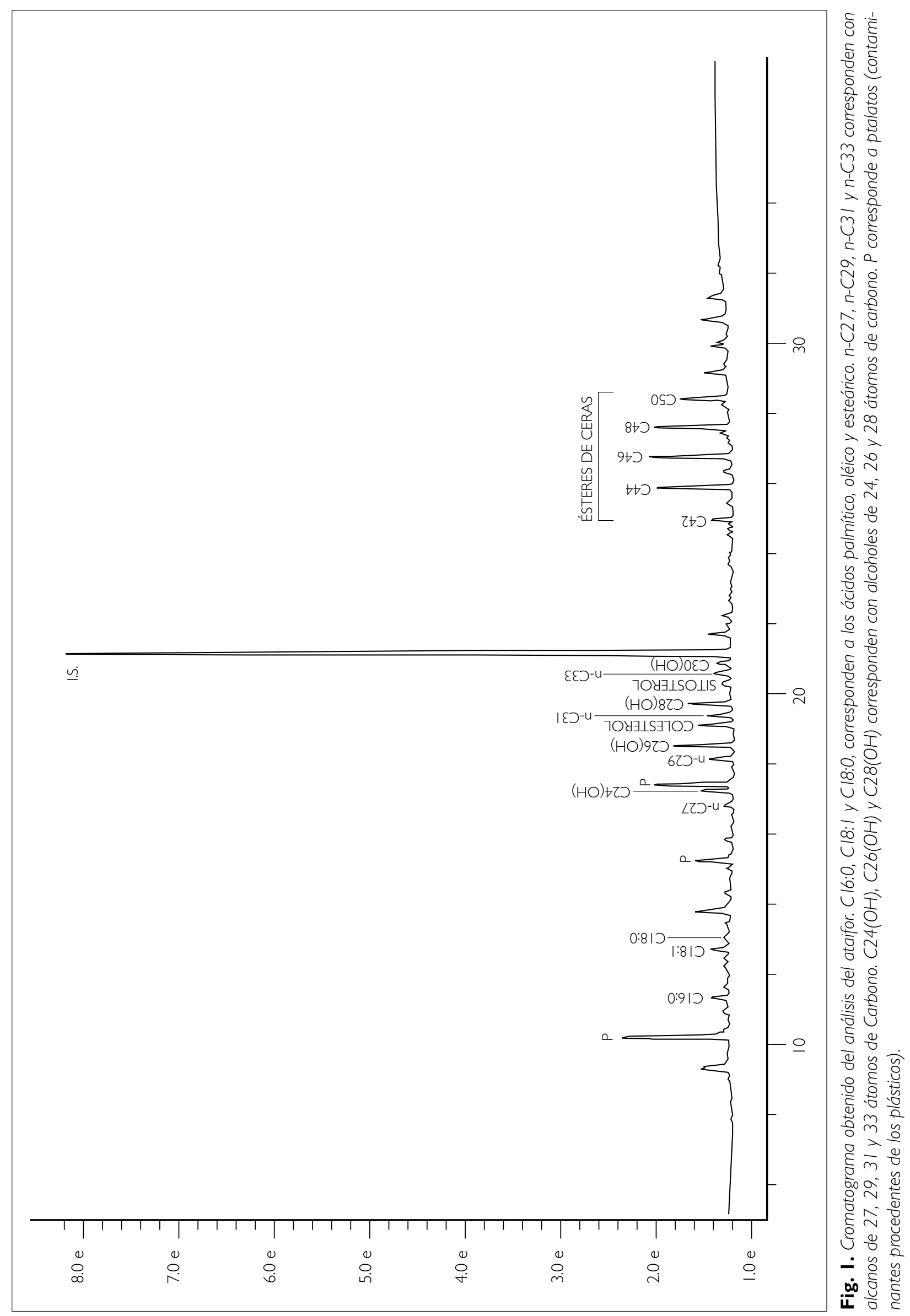

